\title{
Effects of Aging on the Molecular Proffle of Cultured Tendon Cells
}

Carrie E. Barnum, Julianne Huegel, Louis J. Soslowsky, Andrew F. Kuntz

McKay Orthopaedic Research Laboratory, University of Pennsylvania, Philadelphia, PA

\section{Introduction}

Rotator cuff tears affect millions each year, with a higher incidence in the elderly. Surgical repair can improve function and reduce pain, but repair failure is common [1].

- Biologic augmentation has been investigated [2-4].

- Autologous biceps cells delivered to the repair site during supraspinatus repair improve healing in juvenile and aged rats, but do not affect healing in adult rats [5].

- Molecular mechanisms behind differential effects are not well understood.

- Objective: to determine differences in the RNA signature of primary tendon-derived cells from the long head of the biceps of juvenile, adult, and aged animals.

- Hypotheses: 1) juvenile cells exhibit a more stem-cell like molecular profile than aged or adult tendon-derived cells, 2) aged cells have increased expression of genes associated with tendon homeostasis and differentiation compared to juvenile cells.

\section{Methods}
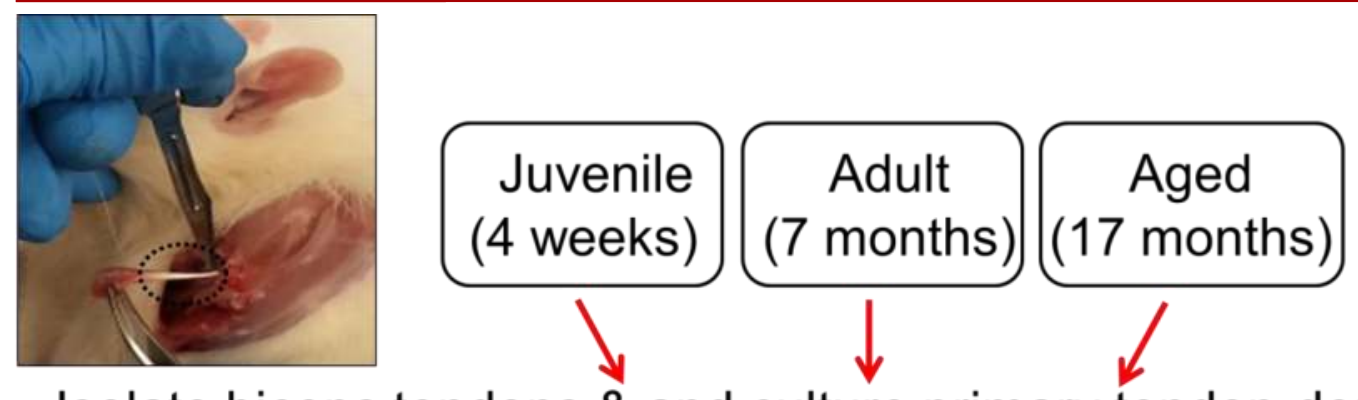

Isolate biceps tendons \& and culture primary tendon-derived cells

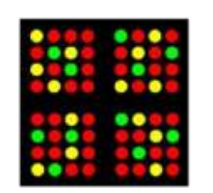

Microarray for RNA gene expression

qPCR validation

27 Fisher (F344) rats used (IACUC approved) across 3 age groups: juvenile, adult, and aged

Cell culture: Animals were euthanized and intraarticular biceps tendons were collected. Biceps tendon cells harvested via tissue morselization and cell migration.

Cell Staining: P1 cells were fixed, permeabilized, stained with Phalloidin and DAPI, and imaged (Leica TCS SP8 Multiphoton Confocal).

RNA Isolation: P1 cells were lysed and homogenized in TRIzol, RNA isolated and purified.

Rat Transcriptome Array and Bioinformatics Analysis: cDNA was synthesized and run (Clariom $^{\mathrm{TM}}$ Rat Transcriptome Array). Bioinformatics processing: Transcriptome Analysis Console (TAC) Software and DAVID ( $n=5 /$ group).

qRT-PCR: qPCR run in quadruplicate using TaqMan assays ( $\mathrm{n}=8 /$ group). Data analyzed with $\triangle \triangle \mathrm{Ct}$ method; expression levels were compared between age groups with one-way ANOVAs and post-hoc Tukey tests.

\section{Results}

Majority of gene expression clustered into six distinct patterns when comparing between ages.

- Majority of differential gene expression between juvenile and aged cells (not shown).
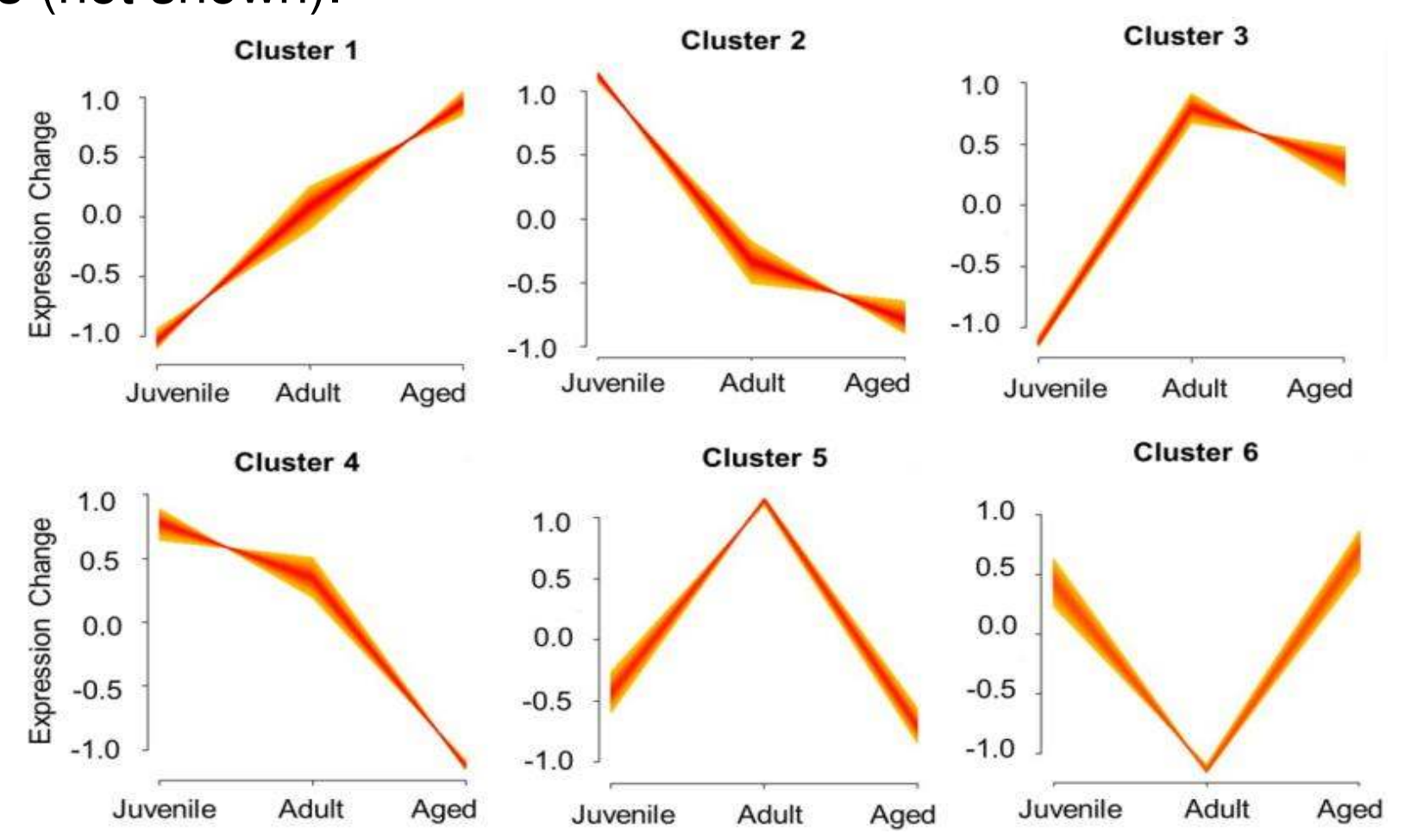

No qualitative differences were observed in cell morphology between age groups.
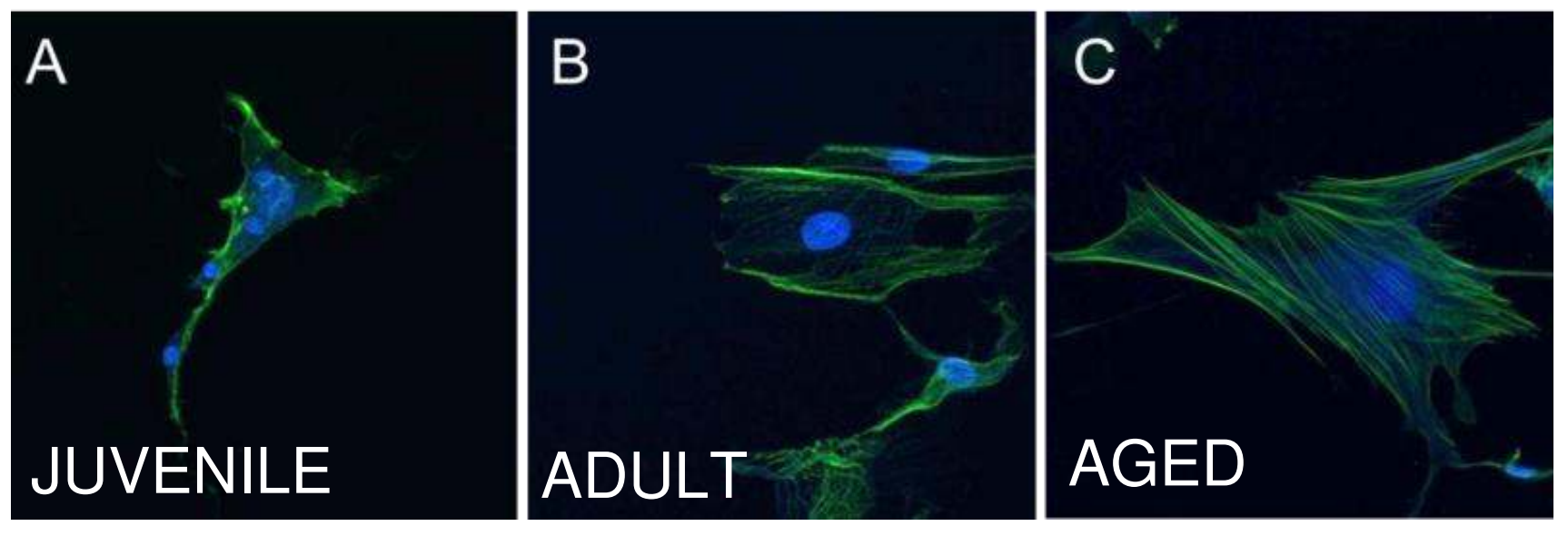

- qPCR confirmed that genes associated with stemness (Fgf10, Postn, Gpnmb, Osr1) are downregulated with age.

- Genes related to inflammation are differentially expressed with age (decreased I/6 and I/6st, increased Cd200 and Cd28).
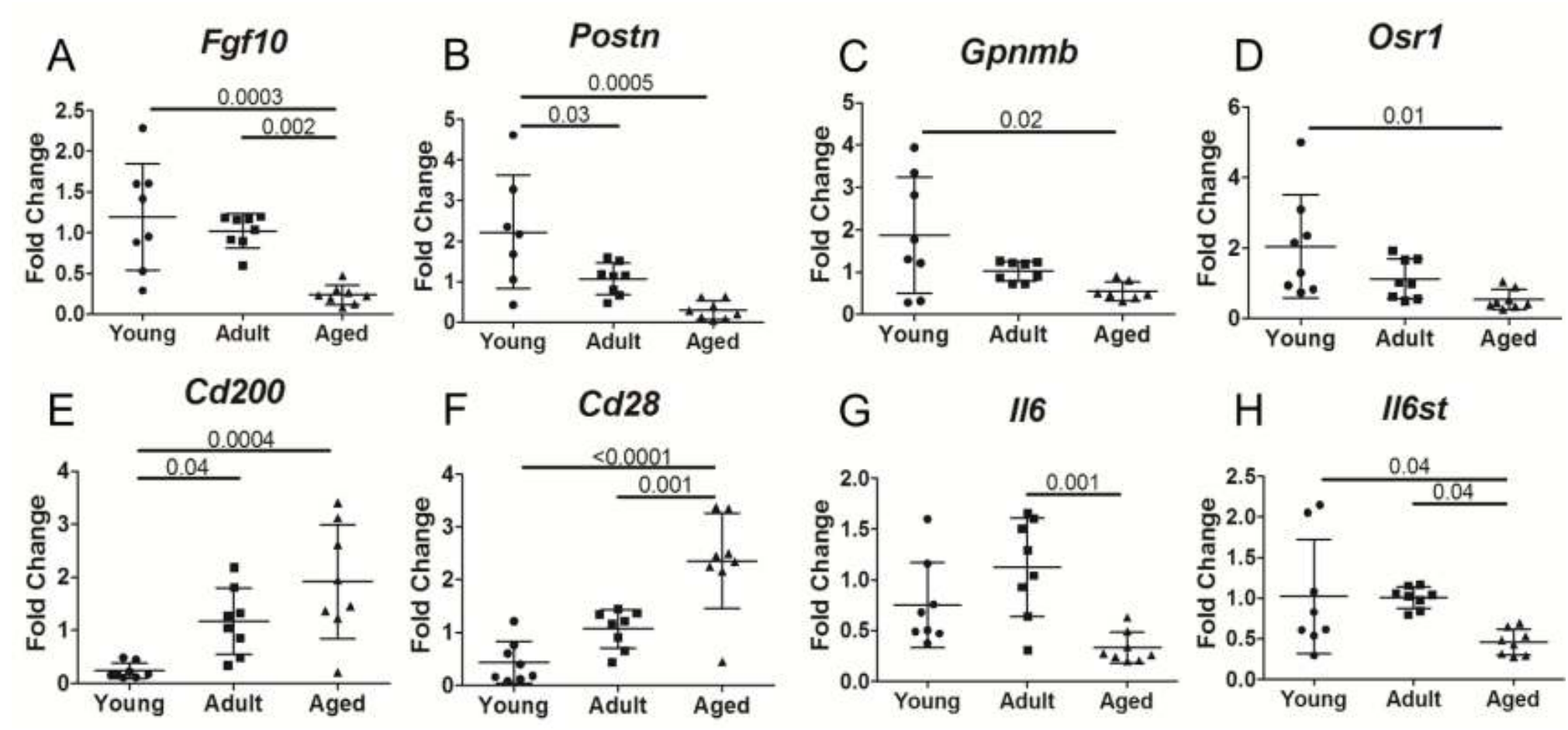

Study supported by the American Shoulder and Elbow Surgeons Research Grant and the Penn Center for Musculoskeletal Disorders (P30 AR069619). We thank Dr. Snehal Shetye for help with cluster analysis. 